\title{
A generalisation of two partition theorems of Andrews
}

\author{
Jehanne Dousse $\|^{1}$ \\ ${ }^{1}$ LIAFA, Université Paris Diderot - Paris 7, 75205 Paris cedex 13, France
}

\begin{abstract}
In 1968 and 1969, Andrews proved two partition theorems of the Rogers-Ramanujan type which generalise Schur's celebrated partition identity (1926). Andrews' two generalisations of Schur's theorem went on to become two of the most influential results in the theory of partitions, finding applications in combinatorics, representation theory and quantum algebra. In this paper we generalise both of Andrews' theorems to overpartitions. The proofs use a new technique which consists in going back and forth from $q$-difference equations on generating functions to recurrence equations on their coefficients.
\end{abstract}

Résumé. En 1968 et 1969, Andrews a prouvé deux identités de partitions du type Rogers-Ramanujan qui généralisent le célèbre théorème de Schur (1926). Ces deux généralisations sont devenues deux des théorèmes les plus importants de la théorie des partitions, avec des applications en combinatoire, en théorie des représentations et en algèbre quantique. Dans ce papier, nous généralisons les deux théorèmes de Andrews aux surpartitions. Les preuves utilisent une nouvelle technique qui consiste à faire des allers-retours entre équations aux $q$-différences sur les séries génératrices et équations de récurrence sur leurs coefficients.

Keywords: integer partitions, overpartitions, q-difference equations, recurrences

\section{Introduction}

A partition of $n$ is a non-increasing sequence of natural numbers whose sum is $n$. An overpartition of $n$ is a partition of $n$ in which the first occurrence of a number may be overlined. For example, there are 14 overpartitions of $4: 4, \overline{4}, 3+1, \overline{3}+1,3+\overline{1}, \overline{3}+\overline{1}, 2+2, \overline{2}+2,2+1+1, \overline{2}+1+1,2+\overline{1}+1, \overline{2}+\overline{1}+1$, $1+1+1+1$ and $\overline{1}+1+1+1$.

In 1926, Schur [Sch26] proved the following partition identity.

Theorem 1.1 (Schur) Let $n$ be a positive integer. Let $D_{1}(n)$ denote the number of partitions of $n$ into distinct parts congruent to 1 or 2 modulo 3 . Let $E_{1}(n)$ denote the number of partitions of $n$ of the form $n=\lambda_{1}+\cdots+\lambda_{s}$ where $\lambda_{i}-\lambda_{i+1} \geq 3$ with strict inequality if $\lambda_{i+1} \equiv 0 \bmod 3$. Then $D_{1}(n)=E_{1}(n)$.

For example, for $n=9$, the partitions counted by $D_{1}(9)$ are $8+1,7+2$ and $5+4$ and the partitions counted by $E_{1}(9)$ are $9,8+1$ and $7+2$. Thus $D_{1}(9)=E_{1}(9)=3$.

\footnotetext{
${ }^{\dagger}$ Email: jehanne.dousse@liafa.univ-paris-diderot.fr 
Several proofs of Schur's theorem have been given using a variety of different techniques such as bijective mappings [Bes91, Bre80], the method of weighted words [AG93], and recurrences [And67, And68b, And71.

Schur's theorem was subsequently generalised to overpartitions by Lovejoy [Lov05], using the method of weighted words. The case $k=0$ corresponds to Schur's theorem.

Theorem 1.2 (Lovejoy) Let $D_{1}(k, n)$ denote the number of overpartitions of $n$ into parts congruent to 1 or 2 modulo 3 with $k$ non-overlined parts. Let $E_{1}(k, n)$ denote the number of overpartitions of $n$ with $k$ non-overlined parts, where parts differ by at least 3 if the smaller is overlined or both parts are divisible by 3, and parts differ by at least 6 if the smaller is overlined and both parts are divisible by 3. Then $D_{1}(k, n)=E_{1}(k, n)$.

Theorem 1.2 was then proved bijectively by Raghavendra and Padmavathamma [RP09], and using $q$-difference equations and recurrences by the author [Dou14].

Andrews extended the ideas of his proofs of Schur's theorem to prove two much more general theorems on partitions with difference conditions [And69, And68a]. But before stating these results in their full generality we need to introduce some notation. Let $A=\{a(1), \ldots, a(r)\}$ be a set of $r$ distinct integers such that $\sum_{i=1}^{k-1} a(i)<a(k)$ for all $1 \leq k \leq r$ and the $2^{r}-1$ possible sums of distinct elements of $A$ are all distinct. We denote this set of sums by $A^{\prime}=\left\{\alpha(1), \ldots, \alpha\left(2^{r}-1\right)\right\}$, where $\alpha(1)<\cdots<\alpha\left(2^{r}-1\right)$. Let us notice that $\alpha\left(2^{k}\right)=a(k+1)$ for all $0 \leq k \leq r-1$ and that any $\alpha$ between $a(k)$ and $a(k+1)$ has largest summand $a(k)$. Let $N$ be a positive integer with $N \geq \alpha\left(2^{r}-1\right)=a(1)+\cdots+a(r)$. We further define $\alpha\left(2^{r}\right)=a(r+1)=N+a(1)$. Let $A_{N}$ denote the set of positive integers congruent to some $a(i) \bmod N,-A_{N}$ the set of positive integers congruent to some $-a(i) \bmod N, A_{N}^{\prime}$ the set of positive integers congruent to some $\alpha(i) \bmod N$ and $-A_{N}^{\prime}$ the set of positive integers congruent to some $-\alpha(i)$ $\bmod N$. Let $\beta_{N}(m)$ be the least positive residue of $m \bmod N$. If $\alpha \in A^{\prime}$, let $w(\alpha)$ be the number of terms appearing in the defining sum of $\alpha$ and $v(\alpha)$ the smallest $a(i)$ appearing in this sum.

To illustrate these notations in the remainder of this paper, it might be useful to consider the example where $a(k)=2^{k-1}$ for $1 \leq k \leq r$ and $\alpha(k)=k$ for $1 \leq k \leq 2^{r}-1$.

We are now able to state Andrews' generalisations of Schur's theorem.

Theorem 1.3 (Andrews) Let $F\left(-A_{N} ; n\right)$ denote the number of partitions of $n$ into distinct parts taken from $-A_{N}$. Let $G\left(-A_{N}^{\prime} ; n\right)$ denote the number of partitions of $n$ into parts taken from $-A_{N}^{\prime}$ of the form $n=\lambda_{1}+\cdots+\lambda_{s}$, such that

$$
\lambda_{i}-\lambda_{i+1} \geq N w\left(\beta_{N}\left(-\lambda_{i}\right)\right)+v\left(\beta_{N}\left(-\lambda_{i}\right)\right)-\beta_{N}\left(-\lambda_{i}\right),
$$

and $\lambda_{s} \geq N\left(w\left(\beta_{N}\left(-\lambda_{s}\right)-1\right)\right.$. Then $F\left(-A_{N} ; n\right)=G\left(-A_{N}^{\prime} ; n\right)$.

Theorem 1.4 (Andrews) Let $D\left(A_{N} ; n\right)$ denote the number of partitions of $n$ into distinct parts taken from $A_{N}$. Let $E\left(A_{N}^{\prime} ; n\right)$ denote the number of partitions of $n$ into parts taken from $A_{N}^{\prime}$ of the form $n=\lambda_{1}+\cdots+\lambda_{s}$, such that

$$
\lambda_{i}-\lambda_{i+1} \geq N w\left(\beta_{N}\left(\lambda_{i+1}\right)\right)+v\left(\beta_{N}\left(\lambda_{i+1}\right)\right)-\beta_{N}\left(\lambda_{i+1}\right) .
$$

Then $D\left(A_{N} ; n\right)=E\left(A_{N}^{\prime} ; n\right)$. 
Not only have Andrews' identities led to a number of important developments in combinatorics [All97. CL06, Yee08] but they also play a natural role in group representation theory [AO91] and quantum algebra [Oh15].

As Schur's theorem generalises to overpartitions and the author was able to generalise the particular case $N=7, r=3, a(1)=1, a(2)=2, a(3)=4$ of Theorems 1.3 and 1.4 in [Dou14], it was interesting to see whether it was possible to extend Andrews' theorems in their full generality to overpartitions. We answer this question by proving the following.

Theorem 1.5 Let $F\left(-A_{N} ; k, n\right)$ denote the number of overpartitions of $n$ into parts taken from $-A_{N}$, having $k$ non-overlined parts. Let $G\left(-A_{N}^{\prime} ; k, n\right)$ denote the number of overpartitions of $n$ into parts taken from $-A_{N}^{\prime}$ of the form $n=\lambda_{1}+\cdots+\lambda_{s}$, having $k$ non-overlined parts, such that

$$
\begin{gathered}
\lambda_{i}-\lambda_{i+1} \geq N w\left(\beta_{N}\left(-\lambda_{i}\right)-1+\chi\left(\overline{\lambda_{i+1}}\right)\right)+v\left(\beta_{N}\left(-\lambda_{i}\right)\right)-\beta_{N}\left(-\lambda_{i}\right), \\
\lambda_{s} \geq N\left(w\left(\beta_{N}\left(-\lambda_{s}\right)\right)-1\right),
\end{gathered}
$$

where $\chi\left(\overline{\lambda_{i+1}}\right)=1$ if $\lambda_{i+1}$ is overlined and 0 otherwise. Then $F\left(-A_{N} ; k, n\right)=G\left(-A_{N}^{\prime} ; k, n\right)$.

Theorem 1.6 Let $D\left(A_{N} ; k, n\right)$ denote the number of overpartitions of $n$ into parts taken from $A_{N}$, having $k$ non-overlined parts. Let $E\left(A_{N}^{\prime} ; k, n\right)$ denote the number of overpartitions of $n$ into parts taken from $A_{N}^{\prime}$ of the form $n=\lambda_{1}+\cdots+\lambda_{s}$, having $k$ non-overlined parts, such that

$$
\lambda_{i}-\lambda_{i+1} \geq N w\left(\beta_{N}\left(\lambda_{i+1}\right)-1+\chi\left(\overline{\lambda_{i+1}}\right)\right)+v\left(\beta_{N}\left(\lambda_{i+1}\right)\right)-\beta_{N}\left(\lambda_{i+1}\right) .
$$

Then $D\left(A_{N} ; k, n\right)=E\left(A_{N}^{\prime} ; k, n\right)$.

Theorem 1.1 (resp. Theorem 1.2 corresponds to $N=3, r=2, a(1)=1, a(2)=2$ in Theorems 1.3 and 1.4 (resp. Theorems 1.5 and 1.6). Again, the case $k=0$ of Theorem 1.5 (resp. Theorem 1.6) gives Theorem 1.3 (resp. Theorem 1.4 ).

Let us illustrate Theorems 1.5 and 1.6 with examples where $N=7, r=3, a(1)=1, a(2)=2$, $a(3)=4$. For Theorem 1.5 the overpartitions of 8 counted by $G\left(-A_{7}^{\prime} ; k, 8\right)$ are $8, \overline{8}, 5+3$ and $\overline{5}+3$. The overpartitions of 8 into parts congruent to 3,5 or 6 modulo 7 (counted by $F\left(-A_{7} ; k, 8\right)$ ) are $5+3, \overline{5}+3$, $5+\overline{3}$ and $\overline{5}+\overline{3}$. In both cases, we have 1 overpartition with 0 non-overlined parts, 2 overpartitions with 1 non-overlined part, and 1 overpartition with 2 non-overlined parts. For Theorem 1.6 , the overpartitions of 4 counted by $E\left(A_{7}^{\prime} ; k, 4\right)$ are $4, \overline{4}, 3+1, \overline{3}+1,2+2, \overline{2}+2,2+1+1, \overline{2}+1+1,1+1+1+1$ and $\overline{1}+1+1+1$. The overpartitions of 4 into parts congruent to 1,2 or 4 modulo 7 (counted by $D\left(A_{7} ; k, 4\right)$ ) are $4, \overline{4}, 2+2, \overline{2}+2,2+1+1, \overline{2}+1+1,2+\overline{1}+1, \overline{2}+\overline{1}+1,1+1+1+1$ and $\overline{1}+1+1+1$. In both cases, we have 1 overpartition with 0 non-overlined parts, 3 overpartitions with 1 non-overlined part, 3 overpartitions with 2 non-overlined parts, 2 overpartitions with 3 non-overlined parts and 1 overpartition with 4 non-overlined parts.

While the statements of Theorems 1.5 and 1.6 resemble those of Andrews' theorems, the proofs are considerably more intricate and involve a number of new ideas. The proof of Theorem 1.6 is presented in its entirety in the paper [Dou15], while the present paper is devoted to an outline of the proof of Theorem 1.5. which is stated here for the first time. Full details will appear in a future publication. First, we give the recurrence equation satisfied by the generating function for overpartitions enumerated by $G\left(-A_{N}^{\prime} ; k, n\right)$ having their largest part $\leq m$, using some combinatorial reasoning on the largest part. Then we prove by induction on $r$ that the limit when $m$ goes to infinity of a function satisfying this 
recurrence equation is equal to $\prod_{j=1}^{r} \frac{\left(-q^{N-a(j)} ; q^{N}\right)_{\infty}}{\left(d q^{N-a(j)} ; q^{N}\right)_{\infty}}$, which is the generating function for overpartitions counted by $F\left(-A_{N} ; k, n\right)$. Here we use the classical notation $(a ; q)_{n}=\prod_{j=0}^{n-1}\left(1-a q^{j}\right)$.

The proof of Theorem 1.6 is somewhat similar. First, we give the $q$-differential equation satisfied by the generating function for overpartitions enumerated by $E\left(A_{N}^{\prime} ; k, n\right)$, using some combinatorial reasoning on the smallest part of the overpartition. Then we prove by induction on $r$ that a function satisfying this $q$-difference equation is equal to $\prod_{j=1}^{r} \frac{\left(-q^{a(j)} ; q^{N}\right)_{\infty}}{\left(d q^{a(j)} ; q^{N}\right)_{\infty}}$, which is the generating function for overpartitions counted by $D\left(A_{N} ; k, n\right)$. However, we will not enter into further details here.

\section{The recurrence equation}

In this section, we establish the recurrence equation satisfied by the generating function for overpartitions enumerated by $G\left(-A_{N}^{\prime} ; k, n\right)$ having their largest part $\leq m$.

Let $n, m \in \mathbb{N}^{*}, k \in \mathbb{N}$. Let $\pi_{m}(k, n)$ denote the number of overpartitions counted by $G\left(-A_{N}^{\prime} ; k, n\right)$ such that the largest part is $\leq m$ and overlined. Let $\phi_{m}(k, n)$ denote the number of overpartitions counted by $G\left(-A_{N}^{\prime} ; k, n\right)$ such that the largest part is $\leq m$ and non-overlined. Then $\psi_{m}(k, n):=\pi_{m}(k, n)+$ $\phi_{m}(k, n)$ is the number of overpartitions counted by $G\left(-A_{N}^{\prime} ; k, n\right)$ with largest part $\leq m$.

Lemma 2.1 We have

$$
\begin{aligned}
& \psi_{j N-\alpha(m)}(k, n)-\psi_{j N-\alpha(m+1)}(k, n) \\
& \quad=\psi_{j N-w(\alpha(m)) N-v(\alpha(m))}(k, n-j N+\alpha(m)) \\
& +\psi_{j N-(w(\alpha(m))-1) N-v(\alpha(m))}(k-1, n-j N+\alpha(m)) .
\end{aligned}
$$

Proof: Let us first prove the following equation:

$$
\begin{aligned}
\pi_{j N-\alpha(m)}(k, n) & =\pi_{j N-\alpha(m+1)}(k, n) \\
& +\pi_{j N-w(\alpha(m)) N-v(\alpha(m))}(k, n-j N+\alpha(m)) \\
& +\phi_{j N-(w(\alpha(m))-1) N-v(\alpha(m))}(k, n-j N+\alpha(m)) .
\end{aligned}
$$

We break the overpartitions counted by $\pi_{j N-\alpha(m)}(k, n)$ into two sets : those with largest part $<j N-$ $\alpha(m)$ and those with largest part equal to $j N-\alpha(m)$. The first set is counted by $\pi_{j N-\alpha(m+1)}(k, n)$, and the second by

$$
\pi_{j N-w(\alpha(m)) N-v(\alpha(m))}(k, n-j N+a(m))+\phi_{j N-(w(\alpha(m))-1) N-v(\alpha(m))}(k, n-j N+a(m)) .
$$

To see this, let us consider an overparition $n=\lambda_{1}+\lambda_{2}+\cdots+\lambda_{s}$ counted by $\pi_{j N-\alpha(m)}(k, n)$ with largest part equal to $j N-\alpha(m)$. Now remove its largest part $\lambda_{1}=j N-\alpha(m)$. The number partitioned becomes $n-j N+\alpha(m)$. The largest part was overlined so the number of non-overlined parts is still $k$. If $\lambda_{2}$ was overlined, then we have

$$
\begin{aligned}
\lambda_{2} & \leq \lambda_{1}-w(\alpha(m)) N-v(\alpha(m))+\alpha(m) \\
& \leq j N-w(\alpha(m)) N-v(\alpha(m))
\end{aligned}
$$


and we obtain an overpartition counted by $\pi_{j N-w(\alpha(m)) N-v(\alpha(m))}(k, n-j N+a(m))$. If $\lambda_{2}$ was not overlined, then we have

$$
\begin{aligned}
\lambda_{2} & \leq \lambda_{1}-(w(\alpha(m))-1) N-v(\alpha(m))+\alpha(m) \\
& \leq j N-(w(\alpha(m))-1) N-v(\alpha(m)),
\end{aligned}
$$

and we obtain an overpartition counted by $\phi_{j N-(w(\alpha(m))-1) N-v(\alpha(m))}(k, n-j N+a(m))$.

In the same way we can prove the following

$$
\begin{aligned}
\phi_{j N-\alpha(m)}(k, n) & =\phi_{j N-\alpha(m+1)}(k, n) \\
& +\pi_{j N-w(\alpha(m)) N-v(\alpha(m))}(k-1, n-j N+\alpha(m)) \\
& +\phi_{j N-(w(\alpha(m))-1) N-v(\alpha(m))}(k-1, n-j N+\alpha(m)) .
\end{aligned}
$$

Adding equations (2) and (3) and noting that for all $m, n, k, \pi_{m}(k-1, n)=\phi_{m}(k, n)$ (we can either overline the largest part or not), we obtain equation (1).

We define, for $m \geq 1,|q|<1,|d|<1$,

$$
g_{m}=g_{m}(q, d):=1+\sum_{n=1}^{\infty} \sum_{k=0}^{\infty} \psi_{m}(k, n) q^{n} d^{k}
$$

and for all $0 \leq k \leq r-1$, we set $g_{-m}(q, d)=(-d)^{k}$ for all $k N \leq m \leq(k+1) N$. This definition is consistent with (1) and the condition that $\lambda_{s} \geq N\left(w\left(\beta_{N}\left(-\lambda_{s}\right)\right)-1\right)$.

Thus equation (1) leads to the following recurrence for $\left(g_{m}\right)$ :

$$
\begin{aligned}
g_{j N-\alpha(m)}=g_{j N-\alpha(m+1)} & +q^{j N-\alpha(m)} g_{j N-w(\alpha(m)) N-v(\alpha(m))} \\
& +d q^{j N-\alpha(m)} g_{j N-(w(\alpha(m))-1) N-v(\alpha(m))} .
\end{aligned}
$$

Let $1 \leq k \leq r+1$. Adding equations (4) together for $1 \leq m \leq 2^{k-1}-1$, using the fact that $\alpha\left(2^{k-1}\right)=$ $a(k)$, and after some manipulations in the sums, we obtain the following recurrence

$$
\begin{aligned}
\left(1-d q^{j N-a(k)}\right) g_{j N-a(k)}=g_{j N-a(k+1)} & +q^{N-a(k)} g_{(j-1) N-a(1)} \\
& +q^{N-a(k)}\left(1-q^{(j-1) N}\right) g_{(j-1) N-a(k)} .
\end{aligned}
$$

We want to find the recurrence equation satisfied by $\left(g_{\ell N-a(1)}\right)_{\ell \in \mathbb{N}}$. Before doing so, we must recall some facts about $q$-binomial coefficients defined by

$$
\left[\begin{array}{c}
m \\
r
\end{array}\right]_{q}:=\left\{\begin{array}{l}
\frac{\left(1-q^{m}\right)\left(1-q^{m-1}\right) \ldots\left(1-q^{m-r+1}\right)}{(1-q)\left(1-q^{2}\right) \ldots\left(1-q^{r}\right)} \text { if } 0 \leq r \leq m, \\
0 \text { otherwise. }
\end{array}\right.
$$

They are $q$-analogues of the binomial coefficients and satisfy $q$-analogues of the Pascal triangle identity [Gas90]. 
Proposition 2.2 For all integers $0 \leq r \leq m$,

$$
\begin{gathered}
{\left[\begin{array}{c}
m \\
r
\end{array}\right]_{q}=q^{r}\left[\begin{array}{c}
m-1 \\
r
\end{array}\right]_{q}+\left[\begin{array}{c}
m-1 \\
r-1
\end{array}\right]_{q}} \\
{\left[\begin{array}{c}
m \\
r
\end{array}\right]_{q}=\left[\begin{array}{c}
m-1 \\
r
\end{array}\right]_{q}+q^{m-r}\left[\begin{array}{c}
m-1 \\
r-1
\end{array}\right]_{q} .}
\end{gathered}
$$

As $q \rightarrow 1$ these equations become Pascal's identity.

Now by induction on $k$ and using equation $[5$ we can prove the following.

Lemma 2.3 For $1 \leq k \leq r+1$, we have

$$
\begin{aligned}
& \prod_{j=1}^{k-1}\left(1-d q^{\ell N-a(j)}\right) g_{\ell N-a(1)}=g_{\ell N-a(k)} \\
& +\sum_{j=1}^{k-1}\left(\sum_{m=0}^{k-j-1} d^{m} \sum_{\substack{\alpha<a(k) \\
w(\alpha)=j+m}} q^{\ell N-\alpha}\left((-1)^{m-1} q^{\ell(m-1) N}\left[\begin{array}{c}
j+m-1 \\
m-1
\end{array}\right]_{q^{-N}}+(-1)^{m} q^{\ell m N}\left[\begin{array}{c}
j+m \\
m
\end{array}\right]_{q^{-N}}\right)\right) \\
& \quad \times \prod_{h=1}^{j-1}\left(1-q^{(\ell-h) N}\right) g_{(\ell-j) N-a(1)} .
\end{aligned}
$$

Writing $u_{\ell}:=g_{\ell N-a(1)}$ and setting $k=r+1$ in Lemma 2.3 we obtain the desired recurrence equation

$$
\begin{aligned}
& \prod_{j=1}^{r}\left(1-d q^{\ell N-a(j)}\right) u_{\ell}=u_{\ell-1} \\
+ & \sum_{j=1}^{r}\left(\sum_{m=0}^{r-j} d^{m} \sum_{\substack{\alpha<a(r+1) \\
w(\alpha)=j+m}} q^{\ell N-\alpha}\left((-1)^{m-1} q^{\ell(m-1) N}\left[\begin{array}{c}
j+m-1 \\
m-1
\end{array}\right]_{q^{-N}}+(-1)^{m} q^{\ell m N}\left[\begin{array}{c}
j+m \\
m
\end{array}\right]_{q^{-N}}\right)\right) \\
& \times \prod_{h=1}^{j-1}\left(1-q^{(\ell-h) N}\right) u_{\ell-j},
\end{aligned}
$$

with the initial conditions $u_{-k}=(-d)^{k}$ for all $0 \leq k \leq r-1$.

\section{Evaluating $\lim _{\ell \rightarrow \infty} u_{\ell}$ by induction}

In this section, we evaluate $\lim _{\ell \rightarrow \infty} u_{\ell}$, which is the generating function for partitions counted by $G\left(-A_{N}^{\prime} ; k, n\right)$. To do so, we prove the following theorem by induction on $r$. The idea of the proof is to start from a function satisfying $\left(\operatorname{rec}_{N, r}\right)$ and to do some transformations to obtain a function satisfying $\left(\operatorname{rec}_{N, r-1}\right)$ in order to use the induction hypothesis. 
Theorem 3.1 Let $r$ be a positive integer. Then for every $N \geq \alpha\left(2^{r}-1\right)$, for every sequence $\left(u_{m}\right)_{m \in \mathbb{N}}$ satisfying $\left(\operatorname{rec}_{N, r}\right)$ and the initial condition $u_{0}=1$, we have

$$
\lim _{\ell \rightarrow \infty} u_{\ell}=\prod_{k=1}^{r} \frac{\left(-q^{N-a(k)} ; q^{N}\right)_{\infty}}{\left(d q^{N-a(k)} ; q^{N}\right)_{\infty}} .
$$

Proof: Let us start by the initial case $r=1$. Let $N \geq a(1)$ and $\left(u_{m}\right)$ such that $u_{0}=1$ and

$$
\left(1-d q^{\ell N-a(1)}\right) u_{\ell}=\left(1+q^{\ell N-a(1)}\right) u_{\ell-1}
$$

$\left(\operatorname{rec}_{N, 1}\right)$

Then

$$
u_{\ell}=\frac{\left(-q^{N-a(1)} ; q^{N}\right)_{\ell}}{\left(d q^{N-a(1)} ; q^{N}\right)_{\ell}}
$$

Taking the limit as $\ell$ goes to infinity gives the desired result.

Now assume that Theorem 3.1 is true for some $r-1 \geq 1$. We want to show that it is true for $r$ too. Let $N \geq \alpha\left(2^{r}-1\right)$, and $\left(u_{m}\right)_{m \in \mathbb{N}}$ satisfy $\left(\operatorname{rec}_{N, r}\right)$ and the initial condition $u_{0}=1$.

For all $m$, let

$$
\beta_{m}:=u_{m} \prod_{j=1}^{m} \frac{1-d q^{j N-a(r)}}{1-q^{j N}} .
$$

Then $\beta_{0}=1$ and after some calculation we can show that $\left(\beta_{m}\right)$ satisfies

$$
\begin{aligned}
& \left(1+\sum_{j=1}^{r}\left(d^{j-1} \sum_{\substack{\alpha<a(r) \\
w(\alpha)=j-1 \\
\min (j-1, h-1)}} q^{-\alpha}+d^{j} \sum_{\substack{\alpha<a(r) \\
w(\alpha)=j}} q^{-\alpha}\right)(-1)^{j} q^{j \ell N}\right) \beta_{\ell} \quad\left(\operatorname{rec}_{N, r}^{\prime}\right) \\
& =\beta_{\ell-1}+\sum_{j=1}^{r} \sum_{h=1}^{r} \sum_{k=0}^{\min c_{k, j} b_{h-k, j}(-1)^{h+1} q^{h \ell N} \beta_{\ell-j},}
\end{aligned}
$$

where

$$
c_{k, j}:=q^{-N \frac{k(k+1)}{2}-k a(r)}\left[\begin{array}{c}
j-1 \\
k
\end{array}\right]_{q^{-N}} d^{k}
$$

and

$$
b_{m, j}:=\left(d^{m-1} \sum_{\substack{\alpha<a(r+1) \\
w(\alpha)=j+m-1}} q^{-\alpha}+d^{m} \sum_{\substack{\alpha<a(r+1) \\
w(\alpha)=j+m}} q^{-\alpha}\right)\left[\begin{array}{c}
j+m-1 \\
m-1
\end{array}\right]_{q^{-N}} .
$$

Now let us define for all $|x|<1$,

$$
f(x):=\sum_{m=0}^{\infty} \beta_{m} x^{m} .
$$


Then $f(0)=1$ and

$$
\begin{aligned}
(1-x) f(x)=\sum_{m=1}^{r} & \left(d^{m-1} \sum_{\substack{\alpha<a(r) \\
w(\alpha)=m-1}} q^{-\alpha}+d^{m} \sum_{\substack{\alpha<a(r) \\
w(\alpha)=m}} q^{-\alpha}\right. \\
& \left.+\sum_{j=1}^{r} \sum_{k=0}^{\min (j-1, m-1)} c_{k, j} b_{m-k, j} x^{j} q^{j m N)}\right)(-1)^{m+1} f\left(x q^{m N}\right) .
\end{aligned}
$$

After some calculation and using properties of $q$-binomial coefficients we can show that this can be rewritten as

$$
\begin{aligned}
(1-x) f(x)= & \sum_{m=1}^{r} \sum_{j=0}^{r-1}\left(d^{m-1} \sum_{\substack{\alpha<a(r) \\
w(\alpha)=j+m-1}} q^{-\alpha}+d^{m} \sum_{\substack{\alpha<a(r) \\
w(\alpha)=j+m}} q^{-\alpha}\right)\left[\begin{array}{c}
j+m-1 \\
m-1
\end{array}\right]_{q^{-N}} \\
& \times(-1)^{m+1} x^{j} q^{j m N} \prod_{k=1}^{m}\left(1+x q^{k N-a(r)}\right) f\left(x q^{m N}\right) .
\end{aligned}
$$

It is thus natural to consider the function

$$
g(x):=\frac{f(x)}{\prod_{k=1}^{\infty}\left(1+x q^{k-a(r)}\right)} .
$$

We obtain that $g(0)=1$ and

$$
\begin{aligned}
(1-x) g(x)= & \sum_{m=1}^{r} \sum_{j=0}^{r-1}\left(d^{m-1} \sum_{\substack{\alpha<a(r) \\
w(\alpha)=j+m-1}} q^{-\alpha}+d^{m} \sum_{\substack{\alpha<a(r) \\
w(\alpha)=j+m}} q^{-\alpha}\right)\left[\begin{array}{c}
j+m-1 \\
m-1
\end{array}\right]_{q^{-N}} \\
& \times(-1)^{m+1} x^{j} q^{j m N} g\left(x q^{m N}\right) .
\end{aligned}
$$

Now let us turn back to recurrence equations again by writing $g(x)=: \sum_{k=0}^{\infty} s_{k} x^{k}$. Then $s_{0}=1$ and

$$
\begin{aligned}
& \left(1-q^{\ell N}\right) \prod_{j=1}^{r-1}\left(1-d q^{\ell N-a(j)}\right) s_{\ell}=s_{\ell-1} \\
+ & \sum_{j=1}^{r-1}\left(\sum_{m=0}^{r-j-1} d^{m} \sum_{\substack{\alpha<a(r) \\
w(\alpha)=j+m}} q^{\ell N-\alpha}\left((-1)^{m-1} q^{\ell(m-1) N}\left[\begin{array}{c}
j+m-1 \\
m-1
\end{array}\right]_{q^{-N}}+(-1)^{m} q^{\ell m N}\left[\begin{array}{c}
j+m \\
m
\end{array}\right]_{q^{-N}}\right)\right) s_{\ell-j} .
\end{aligned}
$$

We are now able to get back to $\operatorname{rec}_{N, r-1}$. Let us define

$$
\mu_{\ell}:=\prod_{k=1}^{\ell}\left(1-q^{k} N\right) s_{\ell} .
$$


Then $\mu_{0}=1$ and $\left(\mu_{\ell}\right)$ satisfies

$$
\begin{aligned}
& \prod_{j=1}^{r-1}\left(1-d q^{\ell N-a(j)}\right) \mu_{\ell}=\mu_{\ell-1} \\
+ & \sum_{j=1}^{r-1}\left(\sum_{m=0}^{r-j-1} d^{m} \sum_{\substack{\alpha<a(r) \\
w(\alpha)=j+m}} q^{\ell N-\alpha}\left((-1)^{m-1} q^{\ell(m-1) N}\left[\begin{array}{c}
j+m-1 \\
m-1
\end{array}\right]_{q^{-N}}+(-1)^{m} q^{\ell m N}\left[\begin{array}{c}
j+m \\
m
\end{array}\right]_{q^{-N}}\right)\right) \\
& \times \prod_{h=1}^{j-1}\left(1-q^{(\ell-h) N}\right) \mu_{\ell-j} .
\end{aligned}
$$

We can now use the induction hypothesis. As $\mu_{0}=1$ and $\left(\mu_{m}\right)$ satisfies $\operatorname{rec}_{N, r-1}$, we know that

$$
\lim _{\ell \rightarrow \infty} \mu_{\ell}=\prod_{k=1}^{r-1} \frac{\left(-q^{N-a(k)} ; q^{N}\right)_{\infty}}{\left(d q^{N-a(k)} ; q^{N}\right)_{\infty}}
$$

Therefore by definition of $\left(s_{\ell}\right)$,

$$
\lim _{\ell \rightarrow \infty} s_{\ell}=\frac{1}{\left(q^{N} ; q^{N}\right)_{\infty}} \prod_{k=1}^{r-1} \frac{\left(-q^{N-a(k)} ; q^{N}\right)_{\infty}}{\left(d q^{N-a(k)} ; q^{N}\right)_{\infty}}
$$

We have

$$
\sum_{m=0}^{\infty} \beta_{m} x^{m}=f(x)=\prod_{k=1}^{\infty}\left(1+x q^{k N-a(r)}\right) g(x)=\prod_{k=1}^{\infty}\left(1+x q^{k N-a(r)}\right) \sum_{m=0}^{\infty} s_{m} x^{m} .
$$

We multiply both sides of $(9)$ by $(1-x)$ and we apply Appell's Comparison Theorem [Die57, p. 101]. We obtain

$$
\lim _{\ell \rightarrow \infty} \beta_{\ell}=\prod_{k=1}^{\infty}\left(1+q^{k N-a(r)}\right) \lim _{\ell \rightarrow \infty} s_{\ell}=\frac{\left(-q^{N-a(r)} ; q^{N}\right)_{\infty}}{\left(q^{N} ; q^{N}\right)_{\infty}} \prod_{k=1}^{r-1} \frac{\left(-q^{N-a(k)} ; q^{N}\right)_{\infty}}{\left(d q^{N-a(k)} ; q^{N}\right)_{\infty}} .
$$

Thus by definition of $\left(\beta_{\ell}\right)$, we have

$$
\lim _{\ell \rightarrow \infty} u_{\ell}=\prod_{j=1}^{\infty} \frac{1-q^{j N}}{1-d q^{j N-a(r)}} \lim _{\ell \rightarrow \infty} \beta_{\ell}=\frac{\left(-q^{N-a(r)} ; q^{N}\right)_{\infty}}{\left(d q^{N-a(r)} ; q^{N}\right)_{\infty}} \prod_{k=1}^{r-1} \frac{\left(-q^{N-a(k)} ; q^{N}\right)_{\infty}}{\left(d q^{N-a(k)} ; q^{N}\right)_{\infty}} .
$$

Theorem 3.1 is proved.

Thus $\lim _{\ell \rightarrow \infty} u_{\ell}$, which is the generating function for partitions counted by $G\left(-A_{N}^{\prime} ; k, n\right)$, is equal to $\prod_{k=1}^{r} \frac{\left(\left(-q^{N-a(k)} ; q^{N}\right)_{\infty}\right.}{\left(d q^{N-a(k)} ; q^{N}\right)_{\infty}}$, which is the generating function for partitions counted by $F\left(-A_{N} ; k, n\right)$. This completes the proof of Theorem 1.5 . 


\section{Ideas for future research}

In [CL06], Corteel and Lovejoy proved an even more general theorem of which both of Andrews' theorems are particular cases. It would be interesting to generalise it to overpartitions too, but new techniques might be necessary.

It would also be interesting to see if Theorems 1.5 and 1.6 have connections with representation theory and quantum algebra like Theorem 1.3 and 1.4

\section{Acknowledgements}

The author thanks Jeremy Lovejoy for many valuable comments and suggestions on an earlier version of this paper.

\section{References}

[AG93] K. Alladi and B. Gordon. Generalizations of Schur's partition theorem. Manuscripta Math., 79:113-126, 1993.

[Al197] K. Alladi. Refinements of rogers-ramanujan type identities. In Special functions, q-series and related topics, volume 14 of Fields Inst. Commun., pages 1-35, 1997.

[And67] G. E. Andrews. On Schur's second partition theorem. Glasgow Math. J., 8:127-132, 1967.

[And68a] G. E. Andrews. A new generalization of Schur's second partition theorem. Acta Arith., 14:429_ 434, 1968.

[And68b] G. E. Andrews. On partition functions related to Schur's second partition theorem. Proc. Amer. Math. Soc., 19:441-444, 1968.

[And69] G. E. Andrews. A general theorem on partitions with difference conditions. Amer. J. Math., 91:18-24, 1969.

[And71] G. E. Andrews. On a theorem of Schur and Gleissberg. Archiv der Mathematik, 22:165-167, 1971.

[AO91] G. E. Andrews and J. B. Olsson. Partition identities with an application to group representation theory. J. Reine Angew. Math., 413:198-212, 1991.

[Bes91] C. Bessenrodt. A combinatorial proof of a refinement of the Andrews-Olsson partition identity. European J. Combin., 12:271-276, 1991.

[Bre80] D. M. Bressoud. A combinatorial proof of Schur's 1926 partition theorem. Proc. Amer. Math. Soc., 79:338-340, 1980.

[CL06] S. Corteel and J. Lovejoy. An iterative-bijective approach to generalizations of Schur's theorem. Eur. J. Comb., 27:496-512, 2006.

[Die57] P. Dienes. The Taylor Series. Dover, 1957. 
[Dou14] J. Dousse. On generalizations of partition theorems of Schur and Andrews to overpartitions. Ramanujan J., 35(3):339-360, 2014.

[Dou15] J. Dousse. A generalisation of a partition theorem of Andrews. Monatsh. Math., to appear, 2015.

[Gas90] G. Gasper. Basic Hypergeometric Series. Cambridge Univ. Press, 1990.

[Lov05] J. Lovejoy. A theorem on seven-colored overpartitions and its applications. Int. J. Number Theory, 1:215-224, 2005.

[Oh15] S. Oh. The Andrews-Olsson identity and Bessenrodt insertion algorithm on Young walls. Eur. J. Comb., 43:8-31, 2015.

[RP09] R. Raghavendra and Padmavathamma. Bressoud's generalization of Schur's theorem extension to overpartitions. New Zealand Journal Of Mathematics, 39:25-32, 2009.

[Sch26] I. Schur. Zur additiven zahlentheorie. Sitzungsberichte der Preussischen Akademie der Wissenschaften, pages 488-495, 1926.

[Yee08] A. J. Yee. Alder's conjecture. J. Reine Angew. Math., 616:67-88, 2008. 
